\title{
LIOUVILLE EXTENSIONS OF ARTINIAN SIMPLE MODULE ALGEBRAS
}

\author{
KATSUTOSHI AMANO
}

\begin{abstract}
In the previous paper [AM], the author and Masuoka developed a PicardVessiot theory for module algebras over a cocommutative pointed smooth Hopf algebra $D$. By using the notion of artinian simple (AS) D-module algebras, it generalizes and unifies the standard Picard-Vessiot theories for linear differential and difference equations. The purpose of this paper is to define the notion of Liouville extensions of AS $D$-module algebras and to characterize the corresponding Picard-Vessiot group schemes.
\end{abstract}

\section{INTRODUCTION}

The notion of Liouville extensions of differential fields first appeared in Kolchin's historical work on the Picard-Vessiot theory [K1]. Picard and Vessiot treated linear differential equations which are solvable by quadratures, without explicit definition. Kolchin gave a rigid definition to its meaning. An extension of differential fields (of zero characteristic) is called Liouville iff it contains no new constants and it is obtained by iterating integrations, exponentiations, and algebraic extensions. A Picard-Vessiot extension is Liouville iff the connected component of its differential Galois group is solvable. By the Lie-Kolchin triangularization theorem and others [K1, Ch. I], we can characterize several types of Liouville extensions in matrix theoretical way. For example, a Liouville extension is obtained only by iterating integrations iff its differential Galois group is unipotent (or "anticompact" in [K1]). In case of arbitrary characteristic, Okugawa [O] studied the Picard-Vessiot theory for fields with higher derivations of infinite length and obtained similar results on Liouville extensions.

Liouville extensions of (ordinary, inversive) difference fields were first studied by Franke $[\mathrm{F}]$. But at that time, the Picard-Vessiot theory remained incomplete since it was restricted to field extensions (see [vPS1, p. 2]). The Picard-Vessiot theory for difference equations in modern sense was developed by van der Put and Singer [vPS1], in which

The author profoundly thanks Prof. A. Masuoka for his many advice. Thanks are also due to the referee who helped the author to correct several mistakes in the manuscript. 
solution algebras can have zero divisors. In this context, Hendriks and Singer [HS] studied on Liouville solutions of difference equations with rational function coefficients. They defined the notion of "Liouvillian sequences" and showed that a linear difference equation can be solved in terms of such sequences iff the difference Galois group is solvable. (Moreover, they gave an algorithm to find such Liouville solutions.)

In the previous paper $[\mathrm{AM}]$, the author and Masuoka developed (following Takeuchi's Hopf algebraic approach $[\mathrm{T}]$ ) a Picard-Vessiot theory for module algebras over a cocommutative pointed smooth Hopf algebra $D$, so that $D=D^{1} \# R G$ where $R$ is the base field, $G=G(D)$ is the grouplikes of $D$, and $D^{1}$ is the irreducible component which contains 1. $D^{1}$ is of Birkhoff-Witt type by the smoothness assumption; namely it is spanned by (possibly infinitely many) divided power sequences of infinite length; an infinite sequence $\left\{1=d_{0}, d_{1}, \ldots, d_{n}, \ldots\right\}$ in $D^{1}$ is called a divided power sequence if $\Delta\left(d_{n}\right)=\sum_{i=0}^{n} d_{i} \otimes d_{n-i}$ [S, p. 268]. We observe divided power sequences act on a commutative $D$-module algebra as higher derivations, while $G$ acts as algebra automorphisms. Thus the notion of commutative $D$-module algebras involves fields with higher derivations of infinite length, inversive difference algebras, and moreover Białynicki-Birula's pioneering notion of "fields with operators" [B]. By using affine group schemes instead of algebraic matrix groups, we removed (as in $[\mathrm{T}]$ ) many assumptions from the Picard-Vessiot theory: for example, the assumption that the constant field is algebraically closed can be removed for many of the results.

The purpose of this paper is to generalize the notion of Liouville extensions for such commutative $D$-module algebras and to characterize the corresponding property of affine group schemes. We assume the reader has introductory knowledge on affine group schemes and Hopf algebras. See [M, S] for basic concepts about Hopf algebras. On affine group schemes, we refer to [W]. On the Picard-Vessiot theory, we follow [AM] for our expositions and notations; here we outline it briefly in the next paragraph.

Throughout this paper, all $D$-module algebras are assumed to be commutative unless otherwise stated. A D-module algebra is called artinian simple (AS) iff it is artinian as a ring and simple as a $D$-module algebra (i.e. it has no non-trivial $D$-stable ideal). We observe that if $K$ is a simple $D$-module algebra, then the constants of $K$, defined by $K^{D}=\{a \in K \mid d a=\varepsilon(d) a$ for all $d \in D\}$ where $\varepsilon$ is the counit, is a field. Following Takeuchi $[\mathrm{T}]$, we define the Picard-Vessiot (PV) extensions as follows. An extension $L / K$ of AS $D$-module algebras is called a $P V$ extension iff $L^{D}=K^{D}$ and there 
exist a (necessarily unique) $D$-module subalgebra $A \subset L$ including $K$ such that the total quotient ring of $A$ is equal to $L$ and $H=\left(A \otimes_{K} A\right)^{D}$ generates the left $A$-module $A \otimes_{K} A$ : $A \otimes_{K} A=A \cdot H$. This $A$ (resp., $H$ ) is called the principal algebra (resp., the Hopf algebra) for $L / K$. To indicate these, we also say that $(L / K, A, H)$ is a PV extension. $H$ has a commutative Hopf algebra structure which defines an affine group scheme $\mathbf{G}(L / K)$ $\left(\simeq \operatorname{Aut}_{D, K \text {-alg }}(A)\right)$ over $K^{D}$, called $P V$ group scheme for $L / K . \mathbf{G}(L / K)$ takes part of the Galois groups; in particular the Galois correspondence between intermediate AS $D$ module algebras of $L / K$ and closed subgroup schemes of $\mathbf{G}(L / K)$ holds. In addition, the definition of PV extensions here is compatible with the definition in the sense of Kolchin $[\mathrm{K}]$ and also of van der Put and Singer [vPS1, vPS2] by the characterization theorem [AM, Theorem 4.6].

When we characterize Liouville extensions in terms of affine group schemes, we will meet the following difficulty: the Lie-Kolchin triangularization theorem cannot be extended generally to affine group schemes (see [W, Ch. 10]). Certainly there are gaps between the triangulability and the connected solvability, even if the base field is algebraically closed. So we need some intermediate notions and have to study how they are related each other. In Section 1, we define "Liouville group schemes" so that it is suitable for our main theorem, and study how strong the definition is. An algebraic affine group scheme G over a field $k$ is called ( $k$-)Liouville (cf. [K, p. 374]) iff there exists a normal chain of closed subgroup schemes $\mathbf{G}=\mathbf{G}_{0} \triangleright \mathbf{G}_{1} \triangleright \cdots \triangleright \mathbf{G}_{r}=\{1\}$ such that each $\mathbf{G}_{i-1} / \mathbf{G}_{i}$ $(i=1, \ldots, r)$ is at least one of the following types: finite etale, a closed subgroup scheme of $\mathbf{G}_{\mathrm{a}}$, or a closed subgroup scheme of $\mathbf{G}_{\mathrm{m}}$. When $k$ is algebraically closed, $\mathbf{G}$ is Liouville iff the connected component $\mathbf{G}^{\circ}$ is solvable (Proposition 1.5). But in general it does not holds; we show this fact by examples. For connected affine group schemes, we will see the condition to be Liouville is properly stronger than the solvability but weaker than the triangulability.

Let $L \supset K$ be an inclusion of AS $D$-module algebras. For finitely many elements $x_{1}, \ldots, x_{n} \in L$, let $K\left\langle x_{1}, \ldots, x_{n}\right\rangle$ denote the smallest AS $D$-module subalgebra in $L$ including both $K$ and $x_{1}, \ldots, x_{n} . \quad L / K$ is called $\mathbf{G}_{\mathrm{a}}$-primitive extension (resp., $\mathbf{G}_{\mathrm{m}^{-}}$ primitive extension) iff there is an $x \in L$ such that $d(x) \in K$ for all $d \in D^{+}=$Ker $\varepsilon$ (resp., $x$ is a non-zero divisor of $L$ (which is necessarily invertible) and $d(x) x^{-1} \in K$ for all $d \in D$ ) and $L=K\langle x\rangle$. We say that $L / K$ is a finite etale extension iff $L$ is a separable $K$-algebra in the sense of [DI], i.e. $L$ is a projective $L \otimes_{K} L$-module. $L / K$ is 
called finitely generated if $L=K\left\langle x_{1}, \ldots, x_{n}\right\rangle$ for some $x_{1}, \ldots, x_{n} \in L$. Then we define Liouville extension as such a finitely generated extension $L / K$ that $L^{D}=K^{D}$ and there exists a sequence of AS $D$-module algebras $K=L_{0} \subset L_{1} \subset \cdots \subset L_{r}=L$ such that each $L_{i} / L_{i-1}(i=1, \ldots, r)$ is at least one of the following types: $\mathbf{G}_{\mathrm{a}}$-primitive extension, $\mathbf{G}_{\mathrm{m}}$-primitive extension, or finite etale extension. As our main theorem, we will show in Theorem 2.10 the following:

Theorem. Let $L / K$ be a finitely generated PV extension. Then the following are equivalent:

(a) $L / K$ is a Liouville extension.

(b) There exists a Liouville extension $F / K$ such that $L \subset F$.

(c) $\mathbf{G}(L / K)$ is Liouville.

When $k$ is algebraically closed, these are equivalent to:

(d) $\mathbf{G}(L / K)^{\circ}$ is solvable.

Moreover we will characterize ten types of Liouville extensions just being compatible with [K1, §24-27]; see Definition 2.8, Corollary 2.12 and its following paragraph.

\section{LiOUVILLE GROUP SCHEMES}

Definition 1.1. Let $\mathbf{G}$ be an algebraic affine group scheme over a field $k$.

(1) We say $\mathbf{G}$ is ( $k$-)Liouville (cf. [K, p. 374]) iff there exists a normal chain of closed subgroup schemes

$$
\mathbf{G}=\mathbf{G}_{0} \triangleright \mathbf{G}_{1} \triangleright \cdots \triangleright \mathbf{G}_{r}=\{1\}
$$

such that each $\mathbf{G}_{i-1} / \mathbf{G}_{i}(i=1, \ldots, r)$ is at least one of the following types: finite etale, a closed subgroup scheme of $\mathbf{G}_{\mathrm{a}}$, or a closed subgroup scheme of $\mathbf{G}_{\mathrm{m}}$. In this case, we call (1.1) a Liouville normal chain (LNC).

(2) In (1.1), if each $\mathbf{G}_{i-1} / \mathbf{G}_{i}$ is merely a closed subgroup scheme of $\mathbf{G}_{\mathrm{a}}$ or a closed subgroup scheme of $\mathbf{G}_{\mathrm{m}}$, then we call it a restricted Liouville normal chain (RLNC).

We use the following abbreviation of some types on group schemes which arise as factor group schemes in an LNC: we say $\mathbf{G}$ is of $\mathbf{G}_{\mathrm{a}}$-type (resp., $\mathbf{G}_{\mathrm{m}}$-type) iff it is a closed subgroup scheme of $\mathbf{G}_{\mathrm{a}}$ (resp., $\mathbf{G}_{\mathrm{m}}$ ), and a group scheme of $R L$-type (resp., L-type) means that it is of $\mathbf{G}_{\mathrm{a}}$-type or $\mathbf{G}_{\mathrm{m}}$-type (resp., RL-type or finite etale). Each type is closed under closed subgroups and quotient groups. 
Lemma 1.2. (1) If $\mathbf{G}$ is Liouville (resp., has an RLNC), then every closed subgroup scheme of $\mathbf{G}$ is Liouville (resp., has an RLNC). Especially $\mathbf{G}$ is Liouville iff the connected component $\mathbf{G}^{\circ}$ is Liouville.

(2) Let $\mathbf{H}$ be a normal closed subgroup scheme of $\mathbf{G}$. Then $\mathbf{G}$ is Liouville (resp., has an $R L N C$ ) iff both $\mathbf{H}$ and $\mathbf{G} / \mathbf{H}$ are Liouville (resp., have an RLNC).

(3) If $\mathbf{G}$ is connected Liouville, then $\mathbf{G}$ is solvable.

Proof. First we take an LNC (resp., an RLNC): $\mathbf{G}=\mathbf{G}_{0} \triangleright \mathbf{G}_{1} \triangleright \cdots \triangleright \mathbf{G}_{r}=\{1\}$ in each proof of (1), "only if" part of (2), and (3).

(1) Let $\mathbf{H}$ be a closed subgroup scheme of $\mathbf{G}$ and put $\mathbf{H}_{i}:=\mathbf{H} \cap \mathbf{G}_{i}(i=0, \ldots, r)$. Then we have $\mathbf{H}_{0}=\mathbf{H}$ and $\mathbf{H}_{i}=\mathbf{H}_{i-1} \cap \mathbf{G}_{i}=\operatorname{Ker}\left(\mathbf{H}_{i-1} \rightarrow \mathbf{G}_{i-1} / \mathbf{G}_{i}\right)$ for $i=1, \ldots, r$. It follows that $\mathbf{H}_{i-1} \triangleright \mathbf{H}_{i}$ and $\mathbf{H}_{i-1} / \mathbf{H}_{i}$ is a closed subgroup scheme of $\mathbf{G}_{i-1} / \mathbf{G}_{i}$ for $i=1, \ldots, r$. Therefore $\mathbf{H}=\mathbf{H}_{0} \triangleright \mathbf{H}_{1} \triangleright \cdots \triangleright \mathbf{H}_{r}=\{1\}$ is an LNC (resp., an RLNC).

(2) ("Only if" part.) $\mathbf{H}$ is Liouville (resp., has an RLNC) by (1). Put $\mathbf{F}_{i}:=\mathbf{G}_{i} / \mathbf{H} \cap \mathbf{G}_{i}$ $(i=0, \ldots, r)$. Since each $k\left[\mathbf{F}_{i-1} / \mathbf{F}_{i}\right]$ is a Hopf subalgebra of $k\left[\mathbf{G}_{i-1} / \mathbf{G}_{i}\right]$, each $\mathbf{F}_{i-1} / \mathbf{F}_{i}$ is of L-type (resp., RL-type) for $i=1, \ldots, r$. Then $\mathbf{G} / \mathbf{H}=\mathbf{F}_{0} \triangleright \mathbf{F}_{1} \triangleright \cdots \triangleright \mathbf{F}_{r}=\{1\}$ is an LNC (resp., an RLNC).

("If" part.) Let $\mathbf{G} / \mathbf{H}=\mathbf{F}_{0} \triangleright \mathbf{F}_{1} \triangleright \cdots \triangleright \mathbf{F}_{r}=\{1\}$ be an LNC (resp., an RLNC) and (0) $=I_{0} \subset I_{1} \subset \cdots \subset I_{r}$ the corresponding sequence of Hopf ideals of $k[\mathbf{G} / \mathbf{H}]$. If we put $I_{i}^{\prime}:=k[\mathbf{G}] \cdot I_{i}(i=0, \ldots, r)$, then each $I_{i}^{\prime}$ becomes a Hopf ideal of $k[\mathbf{G}]$. Let $\mathbf{G}_{i}$ be the closed subgroup scheme of $\mathbf{G}$ which corresponds to $I_{i}^{\prime}$. Then we have a normal chain $\mathbf{G}=\mathbf{G}_{0} \triangleright \mathbf{G}_{1} \triangleright \cdots \triangleright \mathbf{H}$ such that $\mathbf{G}_{i-1} / \mathbf{G}_{i} \simeq \mathbf{F}_{i-1} / \mathbf{F}_{i}(i=1, \ldots, r)$. Therefore $\mathbf{G}$ is Liouville (resp., has an RLNC).

(3) We use induction on the least length $r$ of LNC. The case $r=0$ is clear. Let $r>0$. Since $\mathbf{G}$ is connected, $\mathbf{G} / \mathbf{G}_{1}$ is also connected. Then $\mathbf{G} / \mathbf{G}_{1}$ is of RL-type and hence abelian. Therefore $\mathscr{D G}$ (see $[\mathrm{W},(10.1)]$ ) is a connected closed subgroup scheme of $\mathbf{G}_{1}$. By (1) and its proof, $\mathscr{D} \mathbf{G}$ is connected Liouville and has an LNC with length $\leq r-1$. Then $\mathscr{D} \mathbf{G}$ is solvable by the induction hypothesis, concluding the proof.

The converse of (3) above does not hold in general; see the following example:

Example 1.3. (1) A nontrivial anisotropic torus $\mathbf{T}$ is connected solvable but is not Liouville since both $\operatorname{Hom}\left(\mathbf{T}, \mathbf{G}_{\mathrm{m}}\right)$ and $\operatorname{Hom}\left(\mathbf{T}, \mathbf{G}_{\mathrm{a}}\right)$ are trivial.

(2) Let $k$ be the prime field of $\operatorname{ch}(k)=2$ and $H=k[x] /\left\langle x^{4}+x^{2}+x\right\rangle$ with $x$ primitive. Then $H$ is a commutative Hopf algebra and $\mathbf{G}=\operatorname{Spec} H$ is abelian, finite etale, and 
unipotent. The Cartier dual $\mathbf{G}^{*}$ is finite connected of multiplicative type and then solvable. Since $H^{*}$ does not have any nontrivial grouplike, $\operatorname{Hom}\left(\mathbf{G}^{*}, \mathbf{G}_{\mathrm{m}}\right)$ is trivial. Therefore $\mathrm{G}^{*}$ is not Liouville.

Proposition 1.4. Let $\mathbf{G}$ be a connected algebraic affine group scheme over a field $k$. Then $\mathbf{G}$ is Liouville iff $\mathbf{G}$ has an $R L N C$.

Proof. The "if" part is clear. For the "only if" part, we use induction on the least length $r$ of LNC $\mathbf{G}=\mathbf{G}_{0} \triangleright \mathbf{G}_{1} \triangleright \cdots \triangleright \mathbf{G}_{r}=\{1\}$. The case $r=0$ is clear. Let $r>0$ and assume $\mathbf{G}_{1}^{\circ}$ has an RLNC. By the argument in the proof of Lemma $1.2(3)$, we have $\mathbf{G} \triangleright \mathbf{G}_{1}^{\circ}$ and $\mathbf{G} / \mathbf{G}_{1}^{\circ}$ is abelian. Thus the proof can be reduced to the case that $\mathbf{G}$ is connected abelian by Lemma $1.2(2)$.

Let $\mathbf{G}$ be connected abelian and put $H=k[\mathbf{G}]$. Let $H_{\mathrm{u}}\left(=H^{1}\right)$ be the irreducible component of $H$ which contains 1 (see $[\mathrm{M}, \S 5.6]$ ) and $H_{\mathrm{s}}=H / H H_{\mathrm{u}}^{+}$, where $H_{\mathrm{u}}^{+}$denotes the augmentation ideal of $H_{\mathrm{u}}$. Then we have the exact sequence

$$
H_{\mathrm{u}} \longmapsto H \rightarrow H_{\mathrm{s}}
$$

Let $\bar{k}$ denote the algebraic closure of $k$. It is known that $H_{\mathrm{u}} \otimes_{k} \bar{k}$ is also the irreducible component of $H \otimes_{k} \bar{k}$ containing 1 . The exact sequence (1.2) splits over $\bar{k}$ (the Jordan decomposition of $\mathbf{G}_{\bar{k}}[\mathrm{~W},(9.5)]$ ), and $\mathbf{G}_{\mathrm{s}}:=\operatorname{Spec} H_{\mathrm{s}}$ is connected of multiplicative type since $\left(\mathbf{G}_{\mathrm{s}}\right)_{\bar{k}}$ is connected diagonalizable. Put $\mathbf{G}_{\mathrm{u}}:=\operatorname{Spec} H_{\mathrm{u}}\left(=\mathbf{G} / \mathbf{G}_{\mathrm{s}}\right)$; this is unipotent. We see $\mathbf{G}_{\mathbf{u}}$ has an RLNC whose all factor group schemes are of $\mathbf{G}_{\mathrm{a}}$-type (see [W, Ch. 16, Ex. 5]). Then it suffices to show that $\mathbf{G}_{\mathrm{s}}$ has an RLNC. Let $\mathbf{T}$ be a maximal torus of $\mathbf{G}_{\mathbf{s}}$. $\mathbf{T}$ includes no nontrivial anisotropic subtorus since it is Liouville. Hence, by [W, (7.4)], we see $\mathbf{T}$ is a split torus and has an RLNC. Put $\mathbf{H}=\mathbf{G}_{\mathrm{s}} / \mathbf{T}$; this is finite connected, Liouville, and of multiplicative type. Let $\mathbf{H} \triangleright \mathbf{H}_{1} \triangleright \cdots \triangleright \mathbf{H}_{r}=\{1\}$ be an LNC. We see $\mathbf{H} / \mathbf{H}_{1}$ is of $\mathbf{G}_{\mathrm{m}}$-type. Since $\mathbf{H}$ is finite connected, $k[\mathbf{H}]$ is a local algebra of finite dimension. Then its quotient $k\left[\mathbf{H}_{1}\right]$ is also a local algebra of finite dimension and hence $\mathbf{H}_{1}$ is connected. By the induction hypothesis, $\mathbf{H}_{1}$ has an RLNC. Therefore $\mathbf{H}$ also has an RLNC, concluding the proof.

Proposition 1.5. Let $k$ be an algebraically closed field and $\mathbf{G}$ an algebraic affine group scheme over $k$. Then $\mathbf{G}$ is Liouville iff $\mathbf{G}^{\circ}$ is solvable.

Proof. In fact we have proved the "only if" part in Lemma 1.2 over an arbitrary field. For the "if" part, we use induction on the least $m$ such that $\mathscr{D}^{m} \mathbf{G}^{\circ}=\{1\}$. The case $m=0$ is clear. Let $m>0$ and assume that $\mathscr{D} \mathbf{G}^{\circ}$ has an RLNC. By Lemma 1.2 (2), it suffices 
to show that $\mathbf{G}^{\circ} / \mathscr{D} \mathbf{G}^{\circ}$ has an RLNC. Thus the proof can be reduced to the case that $\mathbf{G}$ is (connected) abelian.

Let $\mathbf{G}$ be abelian and take the Jordan decomposition $\mathbf{G}=\mathbf{G}_{\mathrm{s}} \times \mathbf{G}_{\mathrm{u}} \cdot \mathbf{G}_{\mathrm{u}}$ has an RLNC. Since $k$ is algebraically closed, $\mathbf{G}_{\mathbf{s}}$ is diagonalizable and hence has an RLNC. Therefore G has an RLNC.

We observe that the triangulability is certainly stronger than the condition to have an RLNC, even if $k$ is algebraically closed. For example, the group scheme in [W, Ch. 10, Ex. 3] has an RLNC but is not triangulable.

It is known that $\mathbf{G}$ is unipotent iff $\mathbf{G}$ has an RLNC whose all factor group schemes are of $\mathbf{G}_{\mathrm{a}}$-type. We say that $\mathbf{G}$ is $\mathbf{G}_{\mathrm{m}}$-composite iff $\mathbf{G}$ has an RLNC whose all factor group schemes are of $\mathbf{G}_{\mathrm{m}}$-type. When $k$ is algebraically closed and $\mathbf{G}$ corresponds to the affine algebraic group $\mathbf{G}(k)$ in the sense that the Hopf algebra $k[\mathbf{G}]$ representing $\mathbf{G}$ coincides with the coordinate Hopf algebra of $\mathbf{G}(k), \mathbf{G}$ is $\mathbf{G}_{\mathrm{m}}$-composite iff $\mathbf{G}(k)$ is solvable and "quasicompact" in Kolchin's terminology, which implies that each element of $\mathbf{G}(k)$ is diagonalizable $[\mathrm{K} 1, \S 6$, Theorem 2]. In general it is difficult to characterize the condition to be $\mathbf{G}_{\mathrm{m}}$-composite. As was seen above, group schemes of multiplicative type are not necessarily $\mathbf{G}_{\mathrm{m}}$-composite. On the other hand, non-diagonalizable group schemes can be $\mathbf{G}_{\mathrm{m}}$-composite; see the following example.

Example 1.6. Let $k$ be the prime field with $\operatorname{ch}(k)=p>0$ and take the commutative Hopf algebra $H=k[x, y] /\left\langle x^{p}-x, y^{p}-x-y\right\rangle$ with $x, y$ primitive. One sees $\mathbf{G}=\operatorname{Spec} H$ is abelian, finite etale, and unipotent. Hence the Cartier dual $\mathbf{G}^{*}$ is of multiplicative type and connected. We have the RLNC of $\mathbf{G}$ :

$$
k[x] /\left\langle x^{p}-x\right\rangle \longmapsto H \rightarrow k[y] /\left\langle y^{p}-y\right\rangle .
$$

By dualizing this we see that $\mathbf{G}^{*}$ is $\mathbf{G}_{\mathrm{m}}$-composite:

$$
k\left[\mu_{p}\right] \leftarrow H^{*} \leftarrow k\left[\mu_{p}\right] .
$$

The grouplikes of $H^{*}$ is given by

$$
\operatorname{Coalg}_{k}\left(k, H^{*}\right) \simeq \operatorname{Alg}_{k}(H, k)=\left\{(a, b) \in k^{2} \mid a^{p}-a=0, \quad b^{p}-a-b=0\right\}
$$

where Coalg and Alg denote coalgebra maps and algebra maps respectively. Thus we have $\mathbf{G}^{*}$ is not diagonalizable since $p^{2}=\operatorname{dim}_{k} H^{*} \neq p=\# \operatorname{Alg}_{k}(H, k)$. 


\section{LiOUVILLE EXTENSIONS}

Let $D=D^{1} \# R G$ be a cocommutative pointed smooth Hopf algebra over a base field $R$, as in $[\mathrm{AM}]$. See the fifth paragraph in Introduction for an outline of the PicardVessiot theory for artinian simple (AS) $D$-module algebras. Let $L \supset K$ be an inclusion of AS $D$-module algebras. For finitely many elements $x_{1}, \ldots, x_{n} \in L$, let $K\left\langle x_{1}, \ldots, x_{n}\right\rangle$ denote the smallest AS $D$-module subalgebra in $L$ including both $K$ and $x_{1}, \ldots, x_{n}$. If $L=K\left\langle x_{1}, \ldots, x_{n}\right\rangle$ for some $x_{1}, \ldots, x_{n} \in L$, we say that the extension $L / K$ is finitely generated. A PV extension $L / K$ is finitely generated iff its PV group scheme $\mathbf{G}(L / K)$ is an algebraic affine group scheme (see [AM, Corollary 4.8]).

In the following we always assume that $L / K$ is an extension of AS $D$-module algebras such that $L^{D}=K^{D}=: k$.

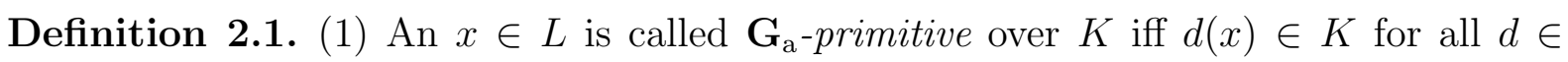
$D^{+}=\operatorname{Ker} \varepsilon$. In this case, we say that $K\langle x\rangle / K$ is a $\mathbf{G}_{\mathrm{a}}$-primitive extension.

(2) An $x \in L$ is called $\mathbf{G}_{\mathrm{m}}$-primitive over $K$ iff $x$ is a non-zero divisor of $L$ and $d(x) x^{-1} \in K$ for all $d \in D$. In this case, we say that $K\langle x\rangle / K$ is a $\mathbf{G}_{\mathrm{m}}$-primitive extension.

(3) We say that $L / K$ is a finite etale extension iff $L$ is a separable $K$-algebra in the sense of [DI], i.e. $L$ is a projective $L \otimes_{K} L$-module. Then, $L$ is necessarily finitely generated (free) as a $K$-module.

For a commutative $K$-algebra $A$, let $\pi_{0}(A)$ denote the union of all separable $K$ subalgebras (in the sense of [DI]) in $A$. Recall that $K$ is the direct product of finitely many (mutually isomorphic) fields $K_{i}$, so that $A$ is the direct product of commutative $K_{i}$-algebra $A_{i}$. Hence $\pi_{0}(A)$ is the direct product of $\pi_{0}\left(A_{i}\right)$, the union of all separable $K_{i}$-subalgebras of $A_{i}$ (see [DI, Ch. II, Proposition 1.13]). It follows that if $A$ is finitely generated as a $K$-algebra, $\pi_{0}(A)$ is the largest separable subalgebra of $A$.

If we take a maximal ideal $P$ of $L$ and put $L^{\prime}=L / P$ and $K^{\prime}=K / P \cap K$, then the following are equivalent:

- $L / K$ is a finite etale extension.

- $L$ is a finitely generated $K$-algebra and $\pi_{0}(L)=L$.

- $L^{\prime} / K^{\prime}$ is a finite separable field extension.

Take a maximal ideal $\mathfrak{p}$ of $K$. We say a finite etale extension $L / K$ is copied (resp., anticopied) iff $L^{\prime}=K^{\prime}$ (resp., $\mathfrak{p} L$ is a maximal ideal of $L$ ); this condition is independent of the choice of $P$ (resp., $\mathfrak{p})$. 
Let $H$ be a commutative $k$-Hopf algebra, $\mathbf{G}=\operatorname{Spec} H$, and $\pi_{0}(H)$ the union of all separable $k$-subalgebras in $H$. Since $\pi_{0}(H)$ is the directed union of all separable $k$-Hopf subalgebras, it is a Hopf subalgebra. The connected component $\mathbf{G}^{\circ}$ of $\mathbf{G}$ is represented by $H / H \pi_{0}(H)^{+}$by definition (see [W, Ch. 6, Ex. 7]).

Proposition 2.2. Let $(L / K, A, H)$ be a PV extension.

(1) $\pi_{0}(A)=\pi_{0}(L)$.

(2) $\pi_{0}(A)$ is the intermediate $A S D$-module algebra which corresponds to $\mathbf{G}(L / K)^{\circ}$ and $\left(\pi_{0}(A) / K, \pi_{0}(A), \pi_{0}(H)\right)$ is a $P V$ extension.

Especially $L / K$ is a finite etale extension iff $\mathbf{G}(L / K)$ is finite etale.

Proof. $(L / K, A, H)$ is a directed union of finitely generated PV extensions $\left(L_{\lambda} / K, A_{\lambda}, H_{\lambda}\right)$ by [AM, Corollary 4.9]. Since $\pi_{0}(L)=\bigcup_{\lambda} \pi_{0}\left(L_{\lambda}\right), \pi_{0}(A)=\bigcup_{\lambda} \pi_{0}\left(A_{\lambda}\right)$, and $\pi_{0}(H)=$ $\bigcup_{\lambda} \pi_{0}\left(H_{\lambda}\right)$, the proof can be reduced to the case that $(L / K, A, H)$ is finitely generated.

(2) By [W, (6.5)] (together with [DI, Ch. II, Proposition 1.13]), we have $\pi_{0}\left(A \otimes_{K} A\right)=$ $\pi_{0}(A) \otimes_{K} \pi_{0}(A)$ and $\pi_{0}\left(A \otimes_{k} H\right)=\pi_{0}\left(A \otimes_{K}\left(K \otimes_{k} H\right)\right)=\pi_{0}(A) \otimes_{K} \pi_{0}\left(K \otimes_{k} H\right)=\pi_{0}(A) \otimes_{k}$ $\pi_{0}(H)$. Thus we obtain an algebra isomorphism $\pi_{0}(A) \otimes_{k} \pi_{0}(H) \stackrel{\sim}{\rightarrow} \pi_{0}(A) \otimes_{K} \pi_{0}(A)$ by restricting the isomorphism $\mu: A \otimes_{k} H \stackrel{\sim}{\rightarrow} A \otimes_{K} A$ in [AM, Proposition 3.4]. This also induces an isomorphism $A \otimes_{k} \pi_{0}(H) \stackrel{\sim}{\rightarrow} A \otimes_{K} \pi_{0}(A)$. Therefore $\theta^{-1}\left(A \otimes_{k} \pi_{0}(H)\right)=\pi_{0}(A)$, where $\theta: A \rightarrow A \otimes_{k} H$ denotes the $H$-comodule structure map of $A$ defined by $\theta(a)=$ $\mu^{-1}(1 \otimes a)$. Since $\theta$ is a $D$-module algebra map, we have that $\pi_{0}(A)$ is a $D$-module subalgebra of $L$.

Then we show that $\pi_{0}(A)$ is the total quotient ring of itself and is an intermediate AS $D$-module algebra of $L / K\left[\mathrm{AM}\right.$, Lemma 2.8]. Let $a$ be a non-zero divisor of $\pi_{0}(A)$. Then it has full support [AM, Corollary 2.9 (i)] and is contained in a separable $K$-subalgebra of $A$ which is a finite product of fields (finite separable over each $K_{i}$ ). Thus $a^{-1}$ is also contained in $\pi_{0}(A)$.

By [AM, Theorem 3.9 (iii)] and its following remarks (see also [T, Theorem 2.9]), we have that $\left(\pi_{0}(A) / K, \pi_{0}(A), \pi_{0}(H)\right)$ is a $\mathrm{PV}$ extension and $\pi_{0}(A)$ is the intermediate AS $D$-module algebra of $L / K$ corresponding to the normal Hopf ideal $H \pi_{0}(H)^{+}$.

(1) By [AM, Proposition 3.13], we may assume that $K$ is a field and $A$ is an integral domain. Put $M=\pi_{0}(A)$. It suffices to show that $M$ is separably closed in $L$. Write $H_{0}=H / H \pi_{0}(H)^{+}$. Then we have an algebra isomorphism $L \otimes_{k} H_{0} \stackrel{\sim}{\rightarrow} L \otimes_{M} A$ since $\left(L / M, A, H_{0}\right)$ is a PV extension [AM, Theorem 3.9 (ii)]. By [W, (6.6)], $\left(L \otimes_{k} H_{0}\right) /$ nilradical is an integral domain. It follows that $\left(L \otimes_{M} L\right) /$ nilradical is also an integral domain since 
it is a localization of $\left(L \otimes_{M} A\right)$ /nilradical by a multiplicative subset not containing zero. Thus $L \otimes_{M} L$ has no nontrivial idempotent. This implies that $M$ is separably closed in $L$. Indeed, let $S$ be an intermediate field of $L / M$ such that $S / M$ is finite separable. If $e \in S \otimes_{M} S\left(\subset L \otimes_{M} L\right)$ is a separability idempotent for $S$ [DI, p. 40], then necessarily $e=1 \otimes 1$. Hence $a \otimes 1-1 \otimes a=0$ in $S \otimes_{M} S$ for all $a \in S$ by the definition of such an idempotent. Therefore $S=M$.

Corollary 2.3. Let $L / K$ be a $P V$ extension. Then $\mathbf{G}(L / K)$ is connected iff $\pi_{0}(L)=K$.

Definition 2.4. Let $\mathscr{G}$ be a finite group and put $H=(k \mathscr{G})^{*}$. We say $L / K$ is a $\mathscr{G}$-extension iff:

(i) $\mathscr{G}$ acts on $L$ as $K$-algebra automorphisms which are $D$-linear, and

(ii) $L / K$ is a right $H$-Galois extension $[\mathrm{M}, \S 8.1]$, or in other words, the map

$$
L \otimes_{K} L \rightarrow \operatorname{Map}(\mathscr{G}, L), \quad x \otimes y \mapsto[\sigma \mapsto x \sigma(y)]
$$

is a bijection.

We easily see that $L / K$ is a $\mathscr{G}$-extension iff $\left(L / K, L,(k \mathscr{G})^{*}\right)$ is a PV extension. If $L / K$ is an anticopied $\mathscr{G}$-extension, then $L^{\prime} / K^{\prime}$ is a Galois extension of fields in ordinary sense such that $\operatorname{Gal}\left(L^{\prime} / K^{\prime}\right)=\mathscr{G}$. Conversely, when $L / K$ is a finite Galois extension of fields, $L / K$ is a $\operatorname{Gal}(L / K)$-extension iff every element of $\operatorname{Gal}(L / K)$ is $D$-linear.

As in [T, (2.5a), (2.5b)], we have the following lemmas:

Lemma 2.5. (1) Let $K\langle x\rangle / K$ be a $\mathbf{G}_{\mathrm{a}}$-primitive extension. Put $A=K[x]$ and $l=$ $1 \otimes_{K} x-x \otimes_{K} 1 \in\left(A \otimes_{K} A\right)^{D}$. Then $(K\langle x\rangle / K, A, k[l])$ is a $P V$ extension with l primitive and the $P V$ group scheme $\mathbf{G}(K\langle x\rangle / K)$ of $\mathbf{G}_{\mathrm{a}}$-type.

(2) Let $K\langle x\rangle / K$ be a $\mathbf{G}_{\mathrm{m}}$-primitive extension. Put $A=K\left[x, x^{-1}\right]$ and $g=x^{-1} \otimes_{K} x \in$ $\left(A \otimes_{K} A\right)^{D}$. Then $\left(K\langle x\rangle / K, A, k\left[g, g^{-1}\right]\right)$ is a $P V$ extension with $g$ grouplike and the $P V$ group scheme $\mathbf{G}(K\langle x\rangle / K)$ of $\mathbf{G}_{\mathrm{m}}$-type.

Proof. (1) $x$ is $\mathbf{G}_{\mathrm{a}}$-primitive iff there exists a $\varphi \in \operatorname{Hom}_{R}(D, K)$ such that $d(x)=\varepsilon(d) x+$ $\varphi(d)$ for all $d \in D$. Then

$$
X=\left(\begin{array}{cc}
1 & 1 \\
x & x+1
\end{array}\right) \in G L_{2}(K\langle x\rangle)
$$

is $G L_{2}$-primitive over $K$ (see $[\mathrm{AM}$, Theorem $4.6(\mathrm{~d})]$ ). In fact,

$$
d X=\left(\begin{array}{cc}
\varepsilon(d) & 0 \\
\varphi(d) & \varepsilon(d)
\end{array}\right) X \quad(d \in D) .
$$


Considering the proof of $[\mathrm{AM}$, Theorem $4.6((\mathrm{~d}) \Rightarrow(\mathrm{a}))]$, we see

$$
Z=\left(X^{-1} \otimes_{K} 1\right)\left(1 \otimes_{K} X\right)=\left(\begin{array}{cc}
1-l & -l \\
l & 1+l
\end{array}\right)
$$

which concludes the proof.

(2) This is equivalent to saying that $x$ is $G L_{1}$-primitive over $K$.

Lemma 2.6. (1) If $l \in\left(L \otimes_{K} L\right)^{D}$ and if $l$ is primitive in the $L$-coring $L \otimes_{K} L$ (see [S1]), then there exists an $x \in L$ such that $l=1 \otimes_{K} x-x \otimes_{K} 1$ and $x$ is $\mathbf{G}_{\mathrm{a}}$-primitive over $K$.

(2) If $g \in\left(L \otimes_{K} L\right)^{D}$ and if $g$ is grouplike in $L \otimes_{K} L$, then there exists a non-zero divisor $x \in L$ such that $g=x^{-1} \otimes_{K} x$ and $x$ is $\mathbf{G}_{\mathrm{m}}$-primitive over $K$.

Proof. (1) Primitive elements in the $L$-coring $L \otimes_{K} L$ are precisely 1-cocycles in the Amitsur complex:

$$
\begin{aligned}
& 0 \rightarrow L \stackrel{\delta_{0}}{\rightarrow} L \otimes_{K} L \stackrel{\delta_{1}}{\rightarrow} L \otimes_{K} L \otimes_{K} L \stackrel{\delta_{2}}{\rightarrow} \cdots, \\
& \delta_{0}(x)=1 \otimes_{K} x-x \otimes_{K} 1, \\
& \delta_{1}\left(\sum x_{i} \otimes_{K} y_{i}\right)=\sum 1 \otimes_{K} x_{i} \otimes_{K} y_{i}-\sum x_{i} \otimes_{K} 1 \otimes_{K} y_{i}+\sum x_{i} \otimes_{K} y_{i} \otimes_{K} 1, \quad \ldots,
\end{aligned}
$$

whose $n$-th cohomology is $H^{n}\left(L / K, \mathbf{G}_{\mathrm{a}}\right)$. But $H^{1}\left(L / K, \mathbf{G}_{\mathrm{a}}\right)=0$ since $L / K$ is a faithfully flat extension (see [W, Ch. 17, Ex. 10]). Then $l \in \operatorname{Ker} \delta_{1}=\operatorname{Im} \delta_{0}$ and hence there exists some $x \in L$ such that $l=1 \otimes_{K} x-x \otimes_{K} 1$. Since $d l=\varepsilon(d) l$ for all $d \in D$, we have $\delta_{0}(d x)=0$ for all $d \in D^{+}$. This implies $d x \in H^{0}\left(L / K, \mathbf{G}_{\mathrm{a}}\right)=K$ for all $d \in D^{+}$.

(2) Grouplike elements in $L \otimes_{K} L$ are precisely 1-cocycles in the complex:

$$
\begin{aligned}
& \{1\} \rightarrow \mathbf{G}_{\mathrm{m}}(L) \stackrel{\delta_{0}}{\rightarrow} \mathbf{G}_{\mathrm{m}}\left(L \otimes_{K} L\right) \stackrel{\delta_{1}}{\rightarrow} \mathbf{G}_{\mathrm{m}}\left(L \otimes_{K} L \otimes_{K} L\right) \stackrel{\delta_{2}}{\rightarrow} \cdots, \\
& \delta_{0}(x)=\left(1 \otimes_{K} x\right)\left(x \otimes_{K} 1\right)^{-1}=x^{-1} \otimes_{K} x, \\
& \delta_{1}\left(\sum x_{i} \otimes_{K} y_{i}\right)=\left(\sum 1 \otimes_{K} x_{i} \otimes_{K} y_{i}\right)\left(\sum x_{i} \otimes_{K} 1 \otimes_{K} y_{i}\right)^{-1}\left(\sum x_{i} \otimes_{K} y_{i} \otimes_{K} 1\right), \quad \ldots,
\end{aligned}
$$

whose $n$-th cohomology is $H^{n}\left(L / K, \mathbf{G}_{\mathrm{m}}\right)$. But $H^{1}\left(L / K, \mathbf{G}_{\mathrm{m}}\right)=\operatorname{Pic}(L / K) \subset \operatorname{Pic}(K)=$ $\{1\}$ since $K$ is a finite product of fields. Then $g \in \operatorname{Ker} \delta_{1}=\operatorname{Im} \delta_{0}$ and hence there exists some $x \in \mathbf{G}_{\mathrm{m}}(L)$ such that $g=x^{-1} \otimes_{K} x$. Since $d g=\varepsilon(d) g$, we have

$$
1 \otimes_{K} d x=d\left(1 \otimes_{K} x\right)=d\left(\left(x \otimes_{K} 1\right) g\right)=d(x) x^{-1} \otimes_{K} x
$$

for all $d \in D$. By multiplying $1 \otimes_{K} x^{-1}$, we have $1 \otimes_{K} d(x) x^{-1}=d(x) x^{-1} \otimes_{K} 1$ for all $d \in D$, which implies $d(x) x^{-1} \in K$ for all $d \in D$.

Proposition 2.7. $L / K$ is a $\mathbf{G}_{\mathrm{a}}$-primitive (resp., $\mathbf{G}_{\mathrm{m}}$-primitive) extension iff $L / K$ is a $P V$ extension and $\mathbf{G}(L / K)$ is of $\mathbf{G}_{\mathrm{a}}$-type (resp., $\mathbf{G}_{\mathrm{m}}$-type). 
Proof. ("Only if" part.) This has been proved in Lemma 2.5.

("If" part.) Let $k[l]$ (resp., $k\left[g, g^{-1}\right]$ ) be the Hopf algebra for $L / K$. By Lemma 2.6, there exists the corresponding $x \in L$. Then $K\langle x\rangle$ is an intermediate AS $D$-module algebra of $L / K$ such that $K\langle x\rangle / K$ is a PV extension. Since the Hopf algebras of $K\langle x\rangle / K$ and $L / K$ coincide, we have $L=K\langle x\rangle$.

Definition 2.8. Let $F / K$ be a finitely generated extension of AS $D$-module algebras such that $F^{D}=K^{D}=k$. We call $F / K$ a Liouville extension iff there exists a sequence of AS $D$-module algebras

$$
K=F_{0} \subset F_{1} \subset \cdots \subset F_{r}=F
$$

such that each $F_{i} / F_{i-1}(i=1, \ldots, r)$ is at least one of the following types: $\mathbf{G}_{\mathrm{a}}$-primitive extension, $\mathbf{G}_{\mathrm{m}}$-primitive extension, or finite etale extension. In this case, the sequence (2.1) is called a Liouville chain. Moreover, $F / K$ is called a Liouville extension of type (j) $(j=1, \ldots, 10)$ iff $F / K$ has a Liouville chain $(2.1)$ such that each extension $F_{i} / F_{i-1}$ $(i=1, \ldots, r)$ is

(1) $\mathbf{G}_{\mathrm{a}}$-primitive, $\mathbf{G}_{\mathrm{m}}$-primitive, or finite etale,

(2) $\mathbf{G}_{\mathrm{a}}$-primitive or $\mathbf{G}_{\mathrm{m}}$-primitive,

(3) $\mathbf{G}_{\mathrm{m}}$-primitive or finite etale,

(4) $\mathbf{G}_{\mathrm{a}}$-primitive or finite etale,

(5) $\mathbf{G}_{\mathrm{a}}$-primitive or a $\mathscr{G}$-extension for a finite solvable group $\mathscr{G}$

(6) $\mathbf{G}_{\mathrm{m}}$-primitive,

(7) $\mathrm{G}_{\mathrm{a}}$-primitive,

(8) finite etale,

(9) a $\mathscr{G}$-extension for a finite solvable group $\mathscr{G}$

(10) trivial (i.e. $F_{i}=F_{i-1}$ ),

respectively. For the list above, we followed [K1, §24]. We observe an anticopied $\mathscr{G}_{-}$ extension for a finite solvable group $\mathscr{G}$ is identified with a Galois extension by radicals and is also a Liouville extension of type (6).

To show the main theorem, we need the following lemma (cf. [K1, 21$]$ ).

Lemma 2.9. Let $L / K$ be a finitely generated $P V$ extension and $F$ an $A S D$-module algebra containing $L$ such that $F^{D}=K^{D}=k$. Take one $t \in F$. Then $L\langle t\rangle / K\langle t\rangle$ is a finitely generated $P V$ extension and $\mathbf{G}(L\langle t\rangle / K\langle t\rangle) \simeq \mathbf{G}(L / K\langle t\rangle \cap L)$. 
Proof. By [AM, Theorem 4.6], there exists a $G L_{n}$-primitive $X=\left(x_{i j}\right) \in G L_{n}(L)$ over $K$ such that $L=K\left\langle x_{i j}\right\rangle$. Since $L\langle t\rangle=K\left\langle t, x_{i j}\right\rangle$, we have that $L\langle t\rangle / K\langle t\rangle$ is a finitely generated PV extension. Write $M=K\langle t\rangle \cap L, Z=\left(X^{-1} \otimes_{M} 1\right)\left(1 \otimes_{M} X\right)=\left(z_{i j}\right)$, and $Z^{-1}=\left(w_{i j}\right)$. Then $H=k\left[z_{i j}, w_{i j}\right]$ becomes the Hopf algebra for $L / M$. Similarly by writing $Z^{\prime}=\left(X^{-1} \otimes_{K\langle t\rangle} 1\right)\left(1 \otimes_{K\langle t\rangle} X\right)=\left(z_{i j}^{\prime}\right)$, and $\left(Z^{\prime}\right)^{-1}=\left(w_{i j}^{\prime}\right)$, we obtain the Hopf algebra $H^{\prime}=k\left[z_{i j}^{\prime}, w_{i j}^{\prime}\right]$ for $L\langle t\rangle / K\langle t\rangle$. It follows that there exists a surjective Hopf algebra map $\varphi: H \rightarrow H^{\prime}, z_{i j} \mapsto z_{i j}^{\prime}$. This implies that $\mathbf{G}(L\langle t\rangle / K\langle t\rangle)$ is a closed subgroup scheme of $\mathbf{G}(L / M)$. Let $I=\operatorname{Ker} \varphi$ be the corresponding Hopf ideal.

$\varphi$ is the restriction (to $H$ ) of the natural map $\tilde{\varphi}: L \otimes_{M} L \rightarrow L\langle t\rangle \otimes_{K\langle t\rangle} L\langle t\rangle$. Since the coideal $I \cdot\left(L \otimes_{M} L\right)$ of $L \otimes_{M} L$, which corresponds to $I$ [AM, Proposition 3.10], is included in $\operatorname{Ker} \tilde{\varphi}$, we have $\left\{a \in L \mid a \otimes_{M} 1-1 \otimes_{M} a \in I \cdot\left(L \otimes_{M} L\right)\right\} \subset\left\{a \in L \mid a \otimes_{M} 1-1 \otimes_{M} a \in\right.$ $\operatorname{Ker} \tilde{\varphi}\}=L \cap K\langle t\rangle=M$. This implies that the intermediate AS $D$-module algebra of $L / M$ which corresponds to $I$ is $M$. Thus $I=0$.

Theorem 2.10. Let $L / K$ be a finitely generated $P V$ extension. Then the following are equivalent:

(a) $L / K$ is a Liouville extension.

(b) There exists a Liouville extension $F / K$ such that $L \subset F$.

(c) $\mathbf{G}(L / K)$ is Liouville.

When $k$ is algebraically closed, these are equivalent to:

(d) $\mathbf{G}(L / K)^{\circ}$ is solvable.

Proof. $((\mathrm{a}) \Rightarrow(\mathrm{b}))$ This is clear.

$((\mathrm{b}) \Rightarrow(\mathrm{c}))$ Take a Liouville chain of $F / K$ :

$$
K=F_{0} \subset F_{1} \subset \cdots \subset F_{r}=F .
$$

We use induction on $r$. The case $r=0$ is obvious. Let $r>0$. Since there are finite $t_{1}, \ldots, t_{s} \in F$ such that $F_{1}=K\left\langle t_{1}, \ldots, t_{s}\right\rangle$, we have that $L\left\langle t_{1}, \ldots, t_{s}\right\rangle / F_{1}$ is a finitely generated PV extension and $\mathbf{G}\left(L\left\langle t_{1}, \ldots, t_{s}\right\rangle / F_{1}\right) \simeq \mathbf{G}\left(L / F_{1} \cap L\right)$ by Lemma 2.9. By the induction hypothesis, $\mathbf{G}\left(L / F_{1} \cap L\right)$ is Liouville. Put $L_{1}=F_{1} \cap L$.

If $F_{1} / K$ is a finite etale extension, then $L_{1} \subset \pi_{0}(L)$. It follows $\mathbf{G}\left(L / L_{1}\right) \supset \mathbf{G}\left(L / \pi_{0}(L)\right)=$ $\mathbf{G}(L / K)^{\circ}$ (Proposition 2.2). Since $\mathbf{G}\left(L / L_{1}\right)$ is Liouville, $\mathbf{G}(L / K)^{\circ}$ is also Liouville and hence (c) holds by Lemma 1.2 . 
If $F_{1} / K$ is a $\mathbf{G}_{\mathrm{a}}$-primitive extension, then one sees that $L_{1} / K$ is also a $\mathbf{G}_{\mathrm{a}}$-primitive extension (see $[\mathrm{T},(2.9 \mathrm{a})])$ ). Hence $\mathbf{G}(L / K) \triangleright \mathbf{G}\left(L / L_{1}\right)$ and $\mathbf{G}(L / K) / \mathbf{G}\left(L / L_{1}\right)=\mathbf{G}\left(L_{1} / K\right)$ is of $\mathbf{G}_{\mathrm{a}}$-type by Proposition 2.7. Therefore (c) holds.

If $F_{1} / K$ is a $\mathbf{G}_{\mathrm{m}}$-primitive extension, then $L_{1} / K$ is also a $\mathbf{G}_{\mathrm{m}}$-primitive extension (see $[\mathrm{T},(2.9 \mathrm{~b})])$. Thus we obtain (c) similarly.

$((\mathrm{c}) \Rightarrow(\mathrm{a}))$ Let $\mathbf{G}(L / K)=\mathbf{G}_{0} \triangleright \mathbf{G}_{1} \triangleright \cdots \triangleright \mathbf{G}_{r}=\{1\}$ be an LNC and $L_{i}(i=0, \ldots, r)$ the intermediate AS $D$-module algebra which corresponds to $\mathbf{G}_{i}$. Then by Proposition 2.2 and by Proposition 2.7, $K=L_{0} \subset L_{1} \subset \cdots \subset L_{r}=L$ is a Liouville chain.

By Proposition 1.4, we have the following.

Corollary 2.11. Let $L / K$ be a finitely generated $P V$ extension. If $L / K$ is a Liouville extension, then there exists a Liouville chain

$$
K=L_{0} \subset \pi_{0}(L)=L_{1} \subset L_{2} \subset \ldots \subset L_{r}=L
$$

such that each $L_{i} / L_{i-1}(i=2, \ldots, r)$ is a $\mathbf{G}_{\mathrm{m}}$-primitive or $\mathbf{G}_{\mathrm{a}}$-primitive extension.

Corollary 2.12. Let $L / K$ be a finitely generated $P V$ extension. Then $L / K$ is (included in) a Liouville extension of type $(j)(j=1, \ldots, 10)$ iff

(1) $\mathbf{G}(L / K)$ is Liouville,

(2) $\mathbf{G}(L / K)$ has an $R L N C$,

(3) $\mathbf{G}(L / K)^{\circ}$ is $\mathbf{G}_{\mathrm{m}}$-composite,

(4) $\mathbf{G}(L / K)^{\circ}$ is unipotent,

(5) $\pi_{0} \mathbf{G}(L / K)$ is finite constant and solvable, and $\mathbf{G}(L / K)^{\circ}$ is unipotent,

(6) $\mathbf{G}(L / K)$ is $\mathbf{G}_{\mathrm{m}}$-composite,

(7) $\mathbf{G}(L / K)$ is unipotent,

(8) $\mathbf{G}(L / K)$ is finite etale,

(9) $\mathbf{G}(L / K)$ is finite constant and solvable,

(10) $\mathbf{G}(L / K)$ is trivial,

respectively.

This corollary can become more explicit when $K$ is a perfect field and $k$ is algebraically closed. In such a case, if $(L / K, A, H)$ is a finitely generated PV extension, then $A \otimes_{K} A$ is reduced (see [W, Ch. 6, Ex. 2]), and so $H$ is reduced. Thus $H$ coincides with the coordinate Hopf algebra of the affine algebraic group $\mathbf{G}(L / K)(k)=\operatorname{Aut}_{D, K \text {-alg }}(L)$ (see $[\mathrm{W},(4.5)])$. There we can do the following replacement on the condition about $\mathbf{G}(L / K)$ :

(1) $\mathbf{G}(L / K)^{\circ}$ is solvable $\left(\Leftrightarrow \mathbf{G}(L / K)^{\circ}\right.$ is triangulable), 
(2) $\mathbf{G}(L / K)$ is solvable,

(3) $\mathbf{G}(L / K)^{\circ}$ is diagonalizable,

(4) $\mathbf{G}(L / K)^{\circ}$ is unipotent,

(5) $\mathbf{G}(L / K)$ is solvable and $\mathbf{G}(L / K)^{\circ}$ is unipotent,

(6) $\mathbf{G}(L / K)(k)$ is solvable and quasicompact (in Kolchin's sense),

(7) $\mathbf{G}(L / K)$ is unipotent,

(8) $\mathbf{G}(L / K)$ is finite constant,

(9) $\mathbf{G}(L / K)$ is finite constant and solvable,

(10) $\mathbf{G}(L / K)$ is trivial.

\section{REFERENCES}

[AM] K. Amano and A. Masuoka, Picard-Vessiot extensions of artinian simple module algebras, J. Algebra 285 (2005), 743-767.

[B] A. Białynicki-Birula, On Galois theory of fields with operators, Amer. J. Math. 84 (1962), 89-109.

[DI] F. DeMeyer and E. Ingraham, "Separable Algebras Over Commutative Rings", LNM 181, Springer, 1971.

[F] C. H. Franke, Picard-Vessiot theory of linear homogeneous difference equations, Trans. Amer. Math. Soc. 108 (1963), 491-515.

[HS] P. A. Hendriks and M. F. Singer, Solving difference equations in finite terms, J. Symbolic Computation 27 (1999), 239-259.

[K1] E. R. Kolchin, Algebraic matric groups and the Picard-Vessiot theory of homogeneous linear ordinary differential equations, Ann. of Math. 49 (1948), 1-42.

[K] E. R. Kolchin, "Differential Algebra and Algebraic Groups", Pure and Applied Mathematics 54, Academic Press, 1973.

[M] S. Montgomery, "Hopf Algebras and Their Actions on Rings", CBMS Reg. Conf. Series 82, Amer. Math. Soc., Providence, 1993.

[O] K. Okugawa, Basic properties of differential fields of an arbitrary characteristic and the PicardVessiot theory, J. Math. Kyoto Univ. 2-3 (1963), 295-322.

[vPS1] M. van der Put and M. F. Singer, "Galois Theory of Difference Equations", LNM 1666, Springer, 1997.

[vPS2] M. van der Put and M. F. Singer, "Galois Theory of Linear Differential Equations", Grundlehren der mathematischen Wissenschaften 328, Springer, 2003.

[S] M. Sweedler, "Hopf Algebras", Benjamin, New York, 1969.

[S1] M. Sweedler, The predual theorem to the Jacobson-Bourbaki theorem, Trans. Amer. Math. Soc. 213 (1975), 391-406.

[T] M. Takeuchi, A Hopf algebraic approach to the Picard-Vessiot theory, J. Algebra 122 (1989), 481-509.

[W] W. C. Waterhouse, "Introduction to Affine Group Schemes", GTM 66, Springer, 1979.

Institute of mathematics, University of TSUKubA, IbARAKi, 305-8571, JAPAN

E-mail address: amano@math.tsukuba.ac.jp 